\title{
IOT Based Weather Reporting System Using Arduino and Node MCU
}

\author{
Meera.S ${ }^{\mathrm{a}, 1}$, Sharmikha Sree.R ${ }^{\mathrm{b}}$, Kalpana R.A ${ }^{\mathrm{c}}$, S.R.Manasvinii ${ }^{\mathrm{c}}$, Haritha. $\mathrm{V}^{\mathrm{d}}$ and \\ Dr. K. Valarmathi ${ }^{\mathrm{e}}$ \\ ${ }^{\mathrm{a}, 1}$ Assistant Professor, Dept of CSE, Sri Sairam Engineering College, Chennai, India \\ ${ }^{b, C, d}$ Student, Dept of CSE, Sri Sairam Engineering College, Chennai, India \\ ${ }^{\mathrm{e}}$ Professor, Dept of CSE, Panimalar Engineering College, Chennai, India
}

\begin{abstract}
Weather is an ever changing phenomenon. It can change in within seconds or milliseconds. Weather was quite difficult to monitor in the days when technology was still primitive. But now it is easy as there are various sensors and computing techniques to not only monitor weather but predict it with high accuracy as well. To simplify the detection process IOT (Internet of things) is employed in the project. As IOT is used Arduino is added to the project along with other sensor to monitor the weather. The data from the sensor is uploaded to an open source web-server. The open server can be used both to get real time data as well as download the data for the prediction process. Prediction is done using machine learning algorithms and training the detected datasets.
\end{abstract}

Keyword: IOT, Machine Learning, Weather, Arduino

\section{Introduction}

Weather reporting system is a monitoring system that displays and relays weather in the server to view. The server also acts a dataset collection center from where the data can be downloaded to feed into the machine learning algorithm. This system works as both a monitoring and predicting system. The technology used for monitoring is IOT i.e. Internet of Things. This incorporates sensors, Arduino and Node MCU Wi-Fi module ESP8622. The sensors detect the climatic changes which is as a data fed to Arduino and this data is inputted to the Node MCU which is then used as module to connect to the server and display the output. The server is open source server where the data can be seen as different feeds and also as a whole in a dashboard where all different feeds can be joined.

\footnotetext{
${ }^{1}$ Meera.S, Assistant Professor, Dept of CSE,Sri Sairam Engineering College, Chennai, India.

E-mail: meera.cse@sairam.edu.in
} 


\section{Related work}

There are various works in this field of study. The main reference of this project is from [1] where the author uses various sensors and Arduino to execute the project. This project also has lot of application from machine learning prediction methods mostly from [2] where the prediction of rainfall and other factors are predicted using multiple linear regression method. This uses target and test method to predict where in the target method historical datasets are fed to train it and in the test method recent datasets are fed to understand the accuracy and compare the output. The paper [3] uses Arduino UNO, temperature, humidity and $\mathrm{CO}$ sensors for detecting carbon monoxide emissions. This project detects the pollution.

In [4] the author uses ESP8266 Node MCU, Temperature, humidity sensor, Arduino UNO, soil moisture sensor to find good temperature for growth of plants and best soil temperature. In [5] the author uses Raspberry Pi, temperature, Humidity sensor PM concentration sensor, Air quality index sensor since the Raspberry Pi is costly and not used for low budget project Arduino is instead replaced in its place. In [6] the author uses API and server and sensors to create a smart alert system for reporting weather. The author uses Internet of things with Arduino and Raspberry pi to execute the project. In [7] the author has used two machine learning algorithms one is multi target regression model and another one is recurrent neural network model using these two models they have predicted rainfall possibilities, temperature and humidity[8-15].

\section{Proposed System Methodology}

The proposed system contains various sensors, Arduino and Node MCU in a setup to read and show the data in sever. As the accuracy of the sensors are high the results obtained are also very accurate. The results are showed in the server by connecting Node MCU to the server. The server is open source and has various options to display the data individually in feeds or together in dashboards. The server also provides an option for downloading the data or feeding it directly into other programs.

\subsection{Prediction using machine learning}

1. The data is collected to feed into the system historical datasets are collected for the target system and the for the test system the datasets from the IOT sensors are used.

2. After the data is collected their features are identified.

3. The training and testing the algorithm is the next step in the process.

4. The training and testing of the datasets is done using multiple linear regression and multi target regression. 


\section{System architecture}
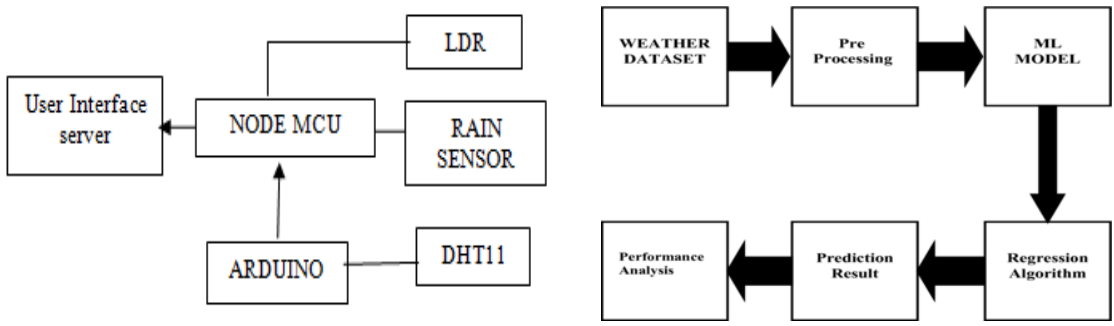

Figure 1. (a) Weather reporting system IOT system architecture (b) Weather reporting system ML system architecture

\subsection{Hardware components}

\subsubsection{Arduino}

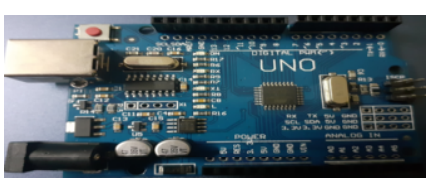

Figure 2. Arduino UNO

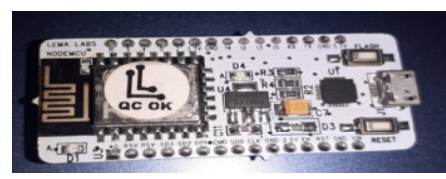

Figure 3. Node MCU

Arduino is the base of the circuit diagram. Arduino is an open-source hardware and software board and board configuration software provided by the same company, It is used in the system to provide power and for data input. Arduino board designs use a variety of micro-processors and micro-controllers. They have series of analog and digital input and output pins. They also have a port to connect to the laptop for power and accessing the COM port for program loading and resetting process.

\subsubsection{Node MCU and DHT11}

Node MCU is a low-cost open source IoT device .nodemcu has 16 general output input pins on its board.it has only one analog pin. EN \&RST pins are the control pins for resetting the microcontroller. Vin pin is used for external power supply.3.3v power can be supplied to $3.3 \mathrm{v}$ pin to power the board. Nodemcu has four pins for SPI communication.

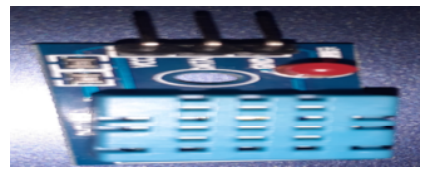

Figure 4. DHT11 sensor with 4 pins 
Dht11 sensor has 4 pins while the dht11 module has 3pins.dht11 sensor and module works the same .but the only difference is, module has inbuilt filtering capacitor and pull up resistors, whereas for the sensor we have to use them externally if required .using dht11 sensor, we can measure temperature and humidity with an accuracy of \pm 1 ${ }^{\circ} \mathrm{C}$ and $\%$ respectively.

\subsubsection{LDR}

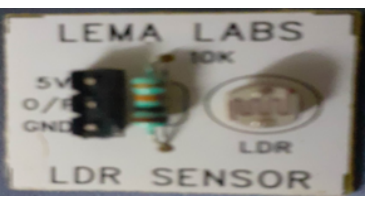

Figure 5. LDR sensor light detector

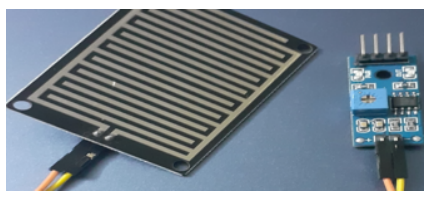

Figure 6. Raindrop sensor and controller

A Light Dependent Resistor also known as LDR is a device that are light-sensitive devices, whose resistivity is a function of the incident electromagnetic radiation. They are also called as photo conductive cells or simply photocells. They have high resistance and are made up of semiconductor materials. When it has low resistance it allows current to pass hence showing its dark and vice-versa.

\subsubsection{Raindrop sensor}

Raindrop Sensor is a tool used for sensing rain. It has 2 parts one detects analog input and another coverts it to digital. It has four pins, they are VCC, GND, D0, A0. D0 pin is the Digital pin.A0 is an Analog pin.

\section{Result}

\subsection{Sensor implementation}

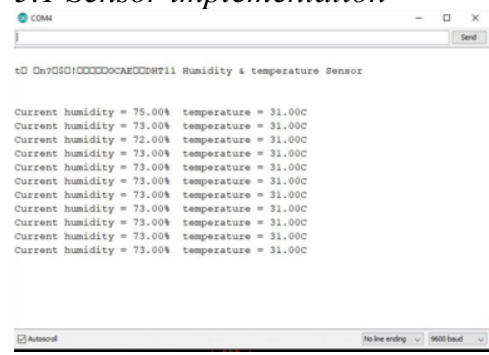

Figure 7. Implementation in Serial monitor of laptop server

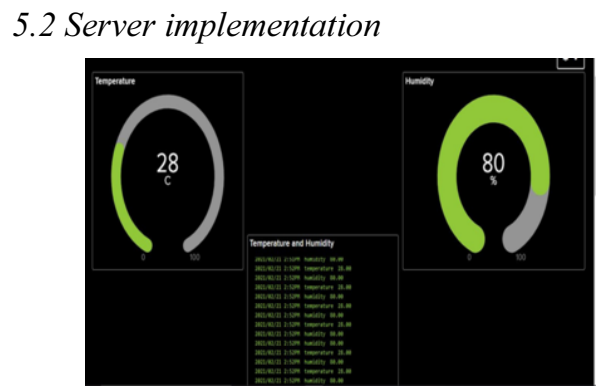

Figure 8. Implementation in open source

\section{Conclusion}

Thus this system deals with both prediction and monitoring of weather. The monitoring is done using Internet of things and prediction is carried out using machine learning 
algorithm and methods such as multiple target regression. This method is very useful in prediction and so it helps in preparing for the future. Be it unexpected natural disaster or unexpected Ain this system helps in preparing early.

\section{References}

[1] Mohit Tiwari, Deepak Narang et al, Weather monitoring system using IOT and cloud computing., International journal of advanced science and technology ,June-2020.

[2] Moulana Mohammed, Prediction Of Rainfall Using Machine Learning Techniques , International Journal of Scientific \& Technology Research Volume 9, Issue 01, January 2020.

[3] Girija C, Hashnalathan, et al, IOT based weather monitoring system, International Journal of engineering research and technology,Apr-2018

[4] Devesh Kumar Srivastava ,IOT Based Weather Reporting System , International Journal of Engineering and Advanced Technology (IJEAT), Volume-9 Issue-5, June 2020.

[5] Ferdian Joe John Joseph, IOT based weather monitoring system for effective analytics., International journal of engineering of and advanced technology APRIL-2019

[6] Yashaswi rahut, Smart weather monitoring and real time alert system using IoT, International Research Journal of Engineering and Technology (IRJET) Volume: 05 Issue: 10 | Oct 2018.

[7] Prathap N Kashyap, Intelligent Weather Forecasting using Machine Learning Techniques, International Research Journal of Engineering and Technology (IRJET )Volume: 07 Issue: 03 | Mar 2020.

[8] Ramya,T.,Dr.Malathi,S.,ratheeksha,G.R. and Dr.V.D.Ambeth Kumar (2014)" Personalized authentication procedure for restricted web service access in mobile phones", Applications of Digital Information and Web Technologies (ICADIWT), 2014, Page(s):69 - 74, Bangalore, India (ISBN:9781-4799-2258-1)

[9] Ambeth Kumar.V.D (2018). Novel Wireless Sensing System For The Welfare Of Sewer Laborers. Healthcare Technology Letters (IET) Volume 5, Issue 4, p. $107-112$.

[10] Ambeth Kumar.V.D, G.Saranya , D.Elangovan, V.RahulChiranjeevi, V.D.Ashok Kumar, "IOT Based Smart Museum Using Wearable Device”, Lecture Notes in Networks and Systems, Vol.55, pp: 33-42, 2018

[11] Ambeth Kumar.V.D and Dr.M.Ramakrishan(2011).Footprint Based Recognition System. in the month of April for the International Journal Communication in Computer and Information System (CCIS) Journal (Springer) Volume 147, Part 3, 358-367, DOI: 10.1007/978-3-642-20573-6_63, April 2011.

[12] Ambeth KumarV.D (2017), "Efficient Routing for Low Rate Wireless Network a Novel Approach", International Journal of Image Mining, Vol. 2, Nos. 3/4, 2017, 2017

[13] Ambeth Kumar.V.D, V.D.Ashok Kumar, Dr.S.Malathi and P.Jagaeedesh, (2014) .Intruder Identification using Footprint Recognition with PCA and SVM Classifiers. for the International Journal of Advanced

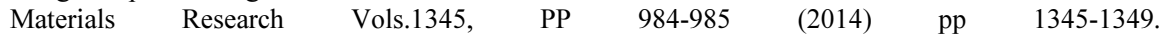
[DOI:10.4028/www.scientific.net/AMR.984-985.1345]

[14] Ambeth Kumar.V.D, G.Gokul, S.Malathi, K.Vengatesan, D.Elangovan, B.Chitra .Implementation Of The Pulse Rhythemic Rate For The Efficient Diagonising Of The Heart Beat. Healthcare Technology Letters (IET) 2019 Apr 17;6(2):48-52.

[15] Ruphitha.S.V et.al. Management of Major Postpartum Haemorrhage by using Zigbee protocol - A Review . 2021 6th International Conference on Inventive Computation Technologies (ICICT) (DOI: 10.1109/ICICT50816.2021.9358757) 